EPJ Web of Conferences 66, 05009 (2014)

DOI: 10.1051/epjconf/ 20146605009

C) Owned by the authors, published by EDP Sciences, 2014

\title{
Search for a permanent EDM using laser cooled radioactive atom
}

\author{
Hirokazu Kawamura ${ }^{1, a}$, S. Ando ${ }^{1}$, T. Aoki ${ }^{1}$, H. Arikawa ${ }^{1}$, S. Ezure ${ }^{1}$, K. Harada ${ }^{1}$, T. Hayamizu ${ }^{1}$, \\ T. Inoue ${ }^{1}$, T. Ishikawa ${ }^{1}$, M. Itoh ${ }^{1}$, K. Kato ${ }^{1}$, T. Kato ${ }^{1, b}$, H. S. Nataraj ${ }^{1, c}$, T. Sato ${ }^{1, d}$, A. Uchiyama ${ }^{1}$, \\ T. Aoki ${ }^{2}$, T. Furukawa ${ }^{3}$, A. Hatakeyama ${ }^{4}$, K. Hatanaka ${ }^{5}$, K. Imai ${ }^{6}$, T. Murakami ${ }^{7}$, Y. Shimizu ${ }^{8}$, \\ T. Wakasa ${ }^{9}$, H. P. Yoshida ${ }^{5}$, and Y. Sakemi ${ }^{1}$ \\ ${ }^{1}$ Cyclotron and Radioisotope Center, Tohoku University, Miyagi 980-8578, Japan \\ ${ }^{2}$ Graduate School of Arts and Sciences, University of Tokyo, Tokyo 153-8902, Japan \\ ${ }^{3}$ Department of Physics, Tokyo Metropolitan University, Tokyo 192-0397, Japan \\ ${ }^{4}$ Department of Applied Physics, Tokyo University of Agriculture and Technology, Tokyo 184-8588, Japan \\ ${ }^{5}$ Research Center for Nuclear Physics, Osaka University, Osaka 567-0047, Japan \\ ${ }^{6}$ Advanced Science Research Center, Japan Atomic Energy Agency, Ibaraki 319-1184, Japan \\ ${ }^{7}$ Department of Physics, Kyoto University, Kyoto 606-8502, Japan \\ ${ }^{8}$ Department of Physics, Tohoku University, Miyagi 980-8578, Japan \\ ${ }^{9}$ Department of Physics, Kyushu University, Fukuoka 812-8581, Japan
}

\begin{abstract}
The search for the electric-dipole moment (EDM) of laser-cooled francium (Fr) atoms could lead to a measurement for the electron EDM. It is predicted that the electron EDM would be enhanced by approximately three orders of magnitude in heavy atoms such as Fr. Laser-cooling and trapping techniques are expected to suppress statistical and systematic errors in precision measurements. The magneto-optical trap was achieved using stable rubidium in a developing factory of laser-cooled radioactive atoms. In light of the results from the rubidium experiments, we found that an upgrade of each apparatus is preferred for Fr trapping.
\end{abstract}

The existence of non-zero electric-dipole moments (EDM) of the elementary particle means the immediate violation of the time-reversal symmetry. Various experiments have been performed in order to search for the new physics beyond the standard model through the precision measurement of EDM. EDM is generally searched in the effect originating from interaction with an external electric field. Therefore, some artifice is required in order to measure the small EDM of a charged particle; in recent years, it has been proposed that a storage-ring facility will allow such experiments. It was found that the EDM of a neutral atom should be measured as the total effect of a nuclear EDM and orbital electrons EDMs [1]. In particular, the EDM of the outermost electron should be greatly enhanced by a nuclear electric field in a hydrogen-like atom.

Conventional experiments used relatively heavy atoms in naturally occurring elements, such as $\mathrm{Cs}$ and $\mathrm{Tl}$. The measurement, which severely restricts the value of the electron EDM in experiments using atoms, actually used a $\mathrm{Tl}$ atomic beam [2]. Experiments using molecules have been recently

\footnotetext{
ae-mail: kawamura@cyric.tohoku.ac.jp

${ }^{b}$ Present address: Progress Technologies Inc.

${ }^{\mathrm{c}}$ Present address: Indian Institute of Technology Roorkee

${ }^{\mathrm{d}}$ Present address: Tokyo Institute of Technology
} 
produced because the electron EDM should be enhanced greatly by an extremely high electric field in polar molecules. In 2011, the experiment using a YbF molecular beam updated the value that had been restricted in the $\mathrm{Tl}$ experiment [3]. These were so-called beam experiments. It is too difficult to arrange an appropriate environment such as uniform electric and magnetic fields for the beam experiment because the measurement requires a very large field. In addition, the velocity of atoms in a beam is so high that it is difficult to improve the measurement's statistical precision since an interaction time between atoms and external fields is quite short. Therefore, it is expected that laser-cooling and trapping techniques can make a breakthrough in precision measurement. The laser cooling is easily applied to an alkali element because of the element's atomic structure. Francium (Fr), which is the heaviest alkali atom, has a large EDM enhancement factor [4]. Unfortunately, Fr is one of the radioactive elements that do not exist stably in nature. Our experimental design consists of the artificial production of Fr using a cyclotron, the application of the laser cooling technique to Fr, and the precise search for Fr EDM, that is, electron EDM. In the proceeding pages, we describe the results obtained in pilot experiments using rubidium and the plans for a Fr trapping experiment.

Laser-cooling and trapping techniques are very useful for precision measurements. A magnetooptical trap (MOT) is one standard technique used to trap a large number of neutral atoms in an ultrahigh vacuum [5]. With this technique, low-velocity atoms can be extracted from thermal atoms and cooled down to millikelvin ranges.

An experiment demonstrated Zeeman slowing to unstable atoms produced by an accelerator and finally a magneto-optical trapping of the unstable atoms [6]. Nuclear reactions produce not only a target isotope, but also considerable radiation, such as neutron and $\gamma$ rays, which negatively affect electronic devices due to irradiation damage. Therefore, some distance from the unstable atom production target to the trapping apparatus is required for radiation protection. The neutral atoms are too difficult to transport efficiently over a long distance; meanwhile, we can transport charged particles relatively easily using electromagnetic fields. This experiment requires Fr production, ion transportation, neutralization and trapping.

A factory of laser-cooled radioactive Fr atoms, shown in Figure 1, has been built to search for the electron EDM at the Cyclotron and Radioisotope Center (CYRIC) at Tohoku University [7]. Fr isotopes are produced through the nuclear fusion reaction between a $100 \mathrm{MeV}$ oxygen beam supplied by an AVF cyclotron and a gold target heated to approximately $1000^{\circ} \mathrm{C}$. When the produced $\mathrm{Fr}$ atom desorbs from the inside of the gold by heat, the Fr is ionized at the surface of the gold whose work function is higher than the ionization potential of Fr. The ionized Fr is extracted by the electric field, which is generated by a voltage of $3 \mathrm{kV}$, and transported over 10 meters using electrostatic lenses. The transported ions are converted into neutral atoms by a neutralizer in the radiation free area. The ions are thermally neutralized at the surface of a hot yttrium metal whose work function is lower than the ionization potential of Fr. The neutralized atoms will be injected into the MOT area and then will be cooled and trapped through the interaction with a magnetic field and lasers.

Although this picture shows a laser-cooled francium factory in its entirety, MOT cannot be appropriated for EDM measurements. MOT requires a quadrupole magnetic field whose intensity is zero at the center; on the other hand, EDM measurement requires a homogeneous magnetic field to assign a quantization axis for atoms. Therefore, the atom trapped in MOT will be delivered into an optical trap that can trap atoms in a potential generated by lasers with no magnetic field. There will be electrodes to apply a high electric field and magnetic coils for the polarization of atoms in the area of the optical trap. The measurement area will equip magnetic shields and magnetometers in order to suppress severe systematic errors originating in the variation of environmental fields [8].

Currently, we are performing experiments in Fr production and trapping in parallel with the development of apparatuses using stable rubidium $(\mathrm{Rb})$, which is one of the alkali elements. The chemical 


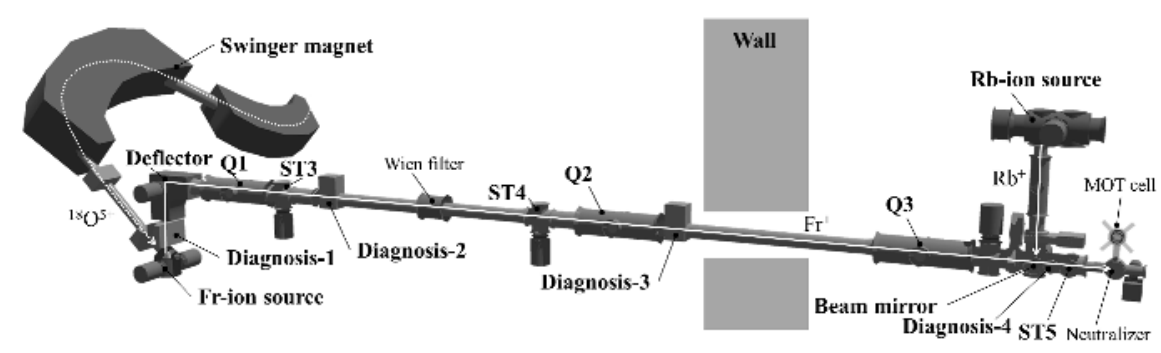

Figure 1. Current project of the laser-cooled Fr factory with a glass cell for MOT. Qx and ST $x$ mean $x$-th electrostatic quadrupole triplet and $x$-th electrostatic double-axis steerer, respectively. Deflector consists of a right-angle deflection electrode and two single-axis steerers. Established structures are indicated by boldface.

properties of $\mathrm{Rb}$ are similar to those of Fr. A cumbersome accelerator is required in order to use Fr. In contrast, $\mathrm{Rb}$ has stable isotopes and is easy to use in the pilot experiments. Therefore, some $\mathrm{Rb}$ sources are installed in our Fr factory. The first source is installed on the thermal ionizer for Fr. The ionizer includes also a source for a $\mathrm{Rb}$ atomic beam and the $\mathrm{Rb}$ is ionized and transported as an ion beam. The second is placed in an orthogonal beam line in front of the neutralizer. An ion source dedicated for $\mathrm{Rb}$ is mounted orthogonally on the mainstream of the Fr beamline. The Rb ionic beam is bent by an electrostatic mirror and merged into the mainstream. This ion source is useful in developing neutralization without transporting the ion over a long distance. The third is an ampule of natural $\mathrm{Rb}$ metals attached to the MOT chamber. $\mathrm{Rb}$ vapor, which enters into the MOT area to heat the ampule, is suitable for the development of the magneto-optical trapping. As described above, $\mathrm{Rb}$ is a requisite element to develop the Fr factory.

The production of Fr ions had been established using a molten gold target. We succeeded in transporting over 10 meters of the Fr ions and the neutralization of the transported Fr [9]. Subsequently, each apparatus development was performed using $\mathrm{Rb}$. The ion transport efficiency was improved to $30 \%$, which is about ten times as much as the value obtained in our previous experiments [7] because the transport conditions were relatively optimized. In addition, the transported $\mathrm{Rb}$ ions were neutralized and trapped in MOT. Past experiments already demonstrated the neutralization and trapping of $\mathrm{Rb}$ in a miniature experimental setup dedicated to $\mathrm{Rb}$ isotopes [7]. This is the first result for $\mathrm{Rb}$ trapping at the laser-cooled Fr factory, which means that the operation test of the whole factory is completed. We estimated the feasibility of the trapping experiment of Fr.

The upgrade of each apparatus is now being continued towards the achievement of Fr MOT. The Fr ion yield was increased by raising the temperature of the gold target. Improving the thermal resistance of the ionizer would lead to an additional increase of the yield. It has also been found that a higher voltage for ion extraction can lead to more efficient transportation. Higher transport efficiency will be achieved by a much higher extraction voltage in a high-temperature environment.

In the ion transport system, a Wien filter and a decelerator will be newly installed. A Wien filter, which is a kind of analyzer of charged particles that uses orthogonal electric and magnetic fields, will be used to select particles other than many isotopes not required for the EDM search produced in the target region. The current beam line consisting of only electrostatic lenses is able to transport any ions with the same charge. In fact, the transported beam surely includes significant components other than the target ions, i.e., Fr and/or Rb. It is considered that the components would be alkali metals included in the insulator and the gold ionized from the fusion target itself, although particle identifications have 
not been carried out. MOT cannot trap different atoms because it uses the resonant between certain isotope and lasers. However, the existence of the components that do not contribute to the trap disturbs the trap as a result of atomic collisions. It was observed that the number of atoms trapped in MOT was decreased by the thermal ion beam [10]. The trapping efficiency will be improved if the Wien filter removes unwanted components.

The incident energy for the neutralizer is an important factor in terms of the trap. The particle implanted onto the surface of the neutralizer metal takes a while to desorb into a vacuum. Unstable atoms cannot ignore the desorption time because of their lifetime. It was observed that the trapping efficiency was increased by slowing the transport velocity due to the differences in implantation depths [11]. On the other hand, the higher extraction voltage is preferable in terms of efficient transportation, as mentioned above. Therefore, we propose to slow down the ions immediately before the injection into the neutralizer. This method is expected to lead to both higher transport efficiency and rapid desorption from the neutralizer.

Our first attempt will employ a method of vapor-cell MOT [12] to trap a Fr isotope. Our current neutralizer is a kind of beam converter that produces an atomic beam from an ion beam. The produced atomic beam is directly trapped in a metal MOT chamber. If transverse and longitudinal cooling is applied to the beam, the trapping efficiency will be drastically increased. On the first try, however, we emphasize the simplicity of vapor-cell MOT that requires no precooling. When the success of Fr MOT provides technical knowledge regarding the cooling and trapping of radioactive atoms, the production of a slow Fr atomic beam will be significantly advanced.

In parallel with the Fr MOT, the optical trapping and EDM measurement system has been developed using $\mathrm{Rb}$. Additionally, an upgrade of the CYRIC facility is planned, such as the system modification of the primary beam transportation and the construction of an electromagnetic shielding room. An experiment searching for the electron EDM will be started when these developments are completed in the future.

The laser-cooled Fr factory has been developed to aid in the search for the electron EDM. At this factory, the ion transportation, neutralization, and MOT succeeded when using a stable Rb isotope. The upgrade of each component of the factory is ongoing for trapping unstable Fr in MOT. On another front, the EDM measurement system has been developed using Rb. We will begin to search for EDM using a Fr isotope within a few years. The authors appreciate the staffs of the CYRIC machine group. This work was mostly supported by Grants-in-Aid for Scientific Research (Nos. 21104005, 10F00023, 23654075 and 23740166) and Tohoku University's Focused Research Project.

\section{References}

[1] M. Pospelov, A. Ritz, Annals of Physics 318, 119-169 (2005)

[2] B.C. Regan, E.D. Commins, C.J. Schmidt and D. DeMille, Phys. Rev. Lett. 88, 071805 (2002)

[3] J.J. Hudson et al., Nature 473, 493-496 (2011)

[4] D. Mukherjee, B.K. Sahoo, H.S. Nataraj and B.P. Das, J. Phys. Chem. A 113, 12549-12557 (2009)

[5] H.J. Metcalf and P.V.D. Straten, Laser cooling and trapping (Springer-Verlag, 1999) 156-164

[6] Z-T. Lu et al., Phys. Rev. Lett. 72, 3791-3794 (1994)

[7] H. Kawamura et al., Nucl. Instr. Meth. B (accepted for publication)

[8] T. Inoue et al., in this proceedings.

[9] T. Sato et al., in this proceedings.

[10] G. Stancari et al., Eur. Phys. J. Special Topics 150, 389-392 (2007)

[11] A.V. Rogachevskiy, Ph. D. thesis, University of Groningen (2007)

[12] C. Monroe, W. Swann, H. Robinson and C. Wieman, Phys. Rev. Lett. 65, 1571-1574 (1990) 